\title{
Young stellar clusters triggered by a density wave in NGC 2997
}

\author{
P. Grosbøl ${ }^{1}$, H. Dottori ${ }^{2}$ and R. Gredel ${ }^{3}$ \\ ${ }^{1}$ European Southern Observatory, Karl-Schwarzschild-Str. 2, 85748 Garching, DE \\ email: pgrosbol@eso.org \\ ${ }^{2}$ Instituto de Física, Univ. Federal do Rio Grande do Sul, Av. Bento Gonçalves 9500, \\ 91501-970 Porto Alegre, RS, BR \\ email: dottori.voy@terra.com.br \\ ${ }^{3}$ Max-Planck Institut für Astronomie, Königstuhl 17, 69117 Heidelberg, DE \\ email: gredel@mpia-hd.mpg.de
}

\begin{abstract}
Bright knots along the arms of grand-design spiral galaxies are frequently seen on near-infrared K-band images. To investigate their nature, low resolution K-band spectra of a string of knots in the southern arm of the grand design, spiral galaxy NGC 2997 were obtained with ISAAC/VLT. Most of the knots show strong $\mathrm{Br} \gamma$ emission while some have $\mathrm{H}_{2}$ and $\mathrm{HeI}$ emission. A few knots show indications of $\mathrm{CO}$ absorption. Their spectra and absolute K magnitudes exceeding -12 mag suggest them to be very compact, young stellar clusters with masses up to $5 \times 10^{4} \mathrm{M}_{\odot}$. The knots' azimuthal distance from the K-band spiral correlates well with their $\operatorname{Br} \gamma$ strength, indicating that they are located inside the co-rotation of the density wave, which triggered them through a large-scale, star-forming front. These relative azimuthal distances suggest an age spread of more than 1.6 Myr, which is incompatible with standard models for an instantaneous star burst. This indicates a more complex star-formation history, such as several bursts or continuous formation.
\end{abstract}

Keywords. galaxies: spiral, galaxies: star clusters, infrared: galaxies, techniques: spectroscopic

\section{Conclusions}

Several K-band emitting knots, aligned along the southern arm of NGC 2997, were observed by Grosbøl et al. (2006). The analysis of the low-resolution, near-infrared spectra gave the following main results:

- their absolute $\mathrm{K}$ magnitudes exceed $-12 \mathrm{mag}$,

- K-band emission spectra are similar to cocoon-enshrouded star forming regions,

- age range is 7-10 Myr and masses are up to $5 \times 10^{4} \mathrm{M}_{\odot}$,

- sizes and masses are similar to that of W49A in our Galaxy,

- alignment with spiral pattern along almost $4 \mathrm{kpc}$ is striking, and

- locations of the knots are consistent with being inside co-rotation of spiral pattern. This suggest a well-synchronized mechanism of piling up the material of which they were formed and for the triggering of star formation similar to the scenario of density wave propagation. Agreement between large-scale, synchronously triggered star formation and knot age differences requires several bursts or continuum star formation instead of a single burst within the knots.

\section{Reference}

Grosbøl, P., Dottori, H. \& Gredel, R. 2006, A\&SA 453, L25 